\title{
Three steps to accelerated expansion
}

\author{
Orest Hrycyna $^{1, *}$ and Marek Szydłowski ${ }^{2,3, * *}$ \\ ${ }^{1}$ Department of Theoretical Physics, Faculty of Philosophy, The John Paul II Catholic University of Lublin, \\ Al. Racławickie 14, 20-950 Lublin, Poland \\ 2 Astronomical Observatory, Jagiellonian University, Orla 171, 30-244 Kraków, Poland \\ ${ }^{3}$ Mark Kac Complex Systems Research Centre, Jagiellonian University, Reymonta 4, 30-059 Kraków, \\ Poland
}

Key words Modified gravity, dark energy theory, scalar field, non-minimal coupling.

PACS 04.50.Kd, 98.80.Cq, 95.36.+x

\begin{abstract}
We study the dynamics of a non-minimally coupled scalar field cosmology with a potential function. We use the framework of dynamical systems theory to investigate all evolutional paths admissible for all initial conditions. Additionally, we assume the presence of barotropic matter and show that the dynamics can be formulated in terms of an autonomous dynamical system. We have found fixed points corresponding to three main stages of the evolution of the universe, namely, radiation, matter and quintessence domination epochs. Using the linearization of the dynamical systems in the vicinity of the critical points we explicitly obtain formulas determining the effective equation of state parameter for the universe at different epochs. In our approach the form of $w(z)$ parametrisation is derived directly from the dynamical equations rather than postulated a priori.
\end{abstract}

Copyright line will be provided by the publisher

There are principally two approaches in searching for the form of the equation of state (EoS) for the current Universe in the accelerating phase of expansion. In the first approach the form of the coefficient $w_{X}(z)$ of the equation of state for dark energy is assumed at the very beginning, usually in the simple linear form with respect to the redshift $z$ or the scale factor $a$ [1,2]. In the second approach the form of the EoS parameterisation is derived from the exact dynamics of the underlying theoretical model [3--7]. In the present paper we realise this idea for the Friedmann-Robertson-Walker (FRW) models filled with a scalar field non-minimally coupled to gravity.

In our study of evolutional scenarios it is natural to use the framework of dynamical system theory because it offers the possibility of investigations of all solutions admissible for all initial conditions. We investigate fixed points of a dynamical system and then linearise the system around them to find exact forms of the EoS parameterisations. Therefore we derive $w(z)$ parameterisations directly from the underlying dynamics of the model.

We assume the spatially flat FRW universe filled with a non-minimally coupled scalar field and barotropic fluid with a general equation of state parameter $w_{m}$, and the action is

$$
S=\frac{1}{2} \int \mathrm{d}^{4} x \sqrt{-g}\left[\frac{1}{\kappa^{2}} R-\varepsilon\left(g^{\mu \nu} \partial_{\mu} \phi \partial_{\nu} \phi+\xi R \phi^{2}\right)-2 U(\phi)\right]+S_{m},
$$

where $\kappa^{2}=8 \pi G, \varepsilon=+1,-1$ corresponds to canonical and phantom scalar field, respectively, and the metric signature is $(-,+,+,+) . S_{m}$ is the action for the barotropic matter part.

We can obtain the dynamical equation for the scalar field from the variation $\delta S / \delta \phi=0$, and energy constraint from the variation $\delta S / \delta g=0$ :

$$
\ddot{\phi}+3 H \dot{\phi}+\xi R \phi+\varepsilon U^{\prime}(\phi)=0, \quad \mathcal{E}=\varepsilon \frac{1}{2} \dot{\phi}^{2}+\varepsilon 3 \xi H^{2} \phi^{2}+\varepsilon 3 \xi H\left(\phi^{2}\right)^{\circ}+U(\phi)+\rho_{m}-\frac{3}{\kappa^{2}} H^{2} .
$$

\footnotetext{
* E-mail: hrycyna@kul.lublin.pl

** E-mail: uoszydlo@cyf-kr.edu.pl
} 
Table 1 The location and eigenvalues of the critical points in twister quintessence scenario

\begin{tabular}{|c|c|c|}
\hline$w_{\text {eff }}$ & location & eigenvalues \\
\hline$\frac{1}{3}$ & $x_{1}^{*}=0, y_{1}^{*}=0,\left(\lambda_{1}^{*}\right)^{2}=\frac{\alpha^{2}}{\varepsilon 6 \xi}$ & $l_{1}=-6 \xi, l_{2}=12 \xi, l_{3}=6 \xi\left(1-3 w_{m}\right)$ \\
$w_{m}$ & $x_{2}^{*}=0, y_{2}^{*}=0, \lambda_{2}^{*}=0$ & $l_{1,3}=-\frac{3}{4}\left(1-w_{m}\right)\left(1 \pm \sqrt{1-\frac{16}{3} \xi \frac{1-3 w_{m}}{\left(1-w_{m}\right)^{2}}}\right), l_{2}=\frac{3}{2}\left(1+w_{m}\right)$ \\
-1 & $x_{3}^{*}=0,\left(y_{3}^{*}\right)^{2}=1, \lambda_{3}^{*}=0$ & $l_{1,3}=-\frac{1}{2}(3 \pm \sqrt{9+\varepsilon 2 \alpha-48 \xi}), l_{2}=-3\left(1+w_{m}\right)$ \\
\hline
\end{tabular}

Then the conservation conditions read

$$
\frac{3}{\kappa^{2}} H^{2}=\rho_{\phi}+\rho_{m}, \quad \dot{H}=-\frac{\kappa^{2}}{2}\left[\left(\rho_{\phi}+p_{\phi}\right)+\rho_{m}\left(1+w_{m}\right)\right],
$$

where the energy density and the pressure of the scalar field are

$$
\begin{array}{r}
\rho_{\phi}=\varepsilon \frac{1}{2} \dot{\phi}^{2}+U(\phi)+\varepsilon 3 \xi H^{2} \phi^{2}+\varepsilon 3 \xi H\left(\phi^{2}\right)^{\circ}, \\
p_{\phi}=\varepsilon \frac{1}{2}(1-4 \xi) \dot{\phi}^{2}-U(\phi)+\varepsilon \xi H\left(\phi^{2}\right)^{\cdot}-\varepsilon 2 \xi(1-6 \xi) \dot{H} \phi^{2}-\varepsilon 3 \xi(1-8 \xi) H^{2} \phi^{2}+2 \xi \phi U^{\prime}(\phi) .
\end{array}
$$

In what follows we introduce the energy phase space variables

$$
x \equiv \frac{\kappa \dot{\phi}}{\sqrt{6} H}, \quad y \equiv \frac{\kappa \sqrt{U(\phi)}}{\sqrt{3} H}, \quad z \equiv \frac{\kappa}{\sqrt{6}} \phi,
$$

which are suggested by the conservation condition $\frac{\kappa^{2}}{3 H^{2}} \rho_{\phi}+\frac{\kappa^{2}}{3 H^{2}} \rho_{m}=\Omega_{\phi}+\Omega_{m}=1$.

The acceleration equation can be written in the form

$$
\dot{H}=-\frac{\kappa^{2}}{2}\left(\rho_{\text {eff }}+p_{\text {eff }}\right)=-\frac{3}{2} H^{2}\left(1+w_{\text {eff }}\right),
$$

where the effective equation of state parameter reads $\left(\lambda=-\frac{\sqrt{6}}{\kappa} \frac{1}{U(\phi)} \frac{\mathrm{d} U(\phi)}{\mathrm{d} \phi}\right)$

$$
\begin{array}{r}
w_{\text {eff }}=\frac{1}{1-\varepsilon 6 \xi(1-6 \xi) z^{2}}\left[-1+\varepsilon(1-6 \xi)\left(1-w_{m}\right) x^{2}+\varepsilon 2 \xi\left(1-3 w_{m}\right)(x+z)^{2}+\right. \\
\left.+\left(1+w_{m}\right)\left(1-y^{2}\right)-\varepsilon 2 \xi(1-6 \xi) z^{2}-2 \xi \lambda y^{2} z\right] .
\end{array}
$$

The dynamical system of the model under consideration takes the form [8,9]

$$
\begin{aligned}
x^{\prime} & =-3 x-12 \xi z+\varepsilon \frac{1}{2} \lambda y^{2}[1-\varepsilon 6 \xi z(x+z)]+\varepsilon 6 \xi(1-6 \xi) x z^{2}+ \\
& +\frac{3}{2}(x+6 \xi z)\left[\varepsilon(1-6 \xi)\left(1-w_{m}\right) x^{2}+\varepsilon 2 \xi\left(1-3 w_{m}\right)(x+z)^{2}+\left(1+w_{m}\right)\left(1-y^{2}\right)\right], \\
y^{\prime} & =-\frac{1}{2} \lambda y\left\{x\left[1-\varepsilon 6 \xi(1-6 \xi) z^{2}\right]+6 \xi y^{2} z\right\}-\varepsilon 12 \xi(1-6 \xi) y z^{2}+ \\
& +\frac{3}{2} y\left[\varepsilon(1-6 \xi)\left(1-w_{m}\right) x^{2}+\varepsilon 2 \xi\left(1-3 w_{m}\right)(x+z)^{2}+\left(1+w_{m}\right)\left(1-y^{2}\right)\right] \\
z^{\prime} & =x\left[1-\varepsilon 6 \xi(1-6 \xi) z^{2}\right] \\
\lambda^{\prime} & =-\lambda^{2}(\Gamma-1) x\left[1-\varepsilon 6 \xi(1-6 \xi) z^{2}\right]
\end{aligned}
$$


where $\Gamma=\frac{U^{\prime \prime}(\phi) U(\phi)}{U^{\prime}(\phi)^{2}}$ and a prime denotes differentiation with respect to time $\tau$ defined as $\frac{\mathrm{d}}{\mathrm{d} \tau}=[1-$ $\left.\varepsilon 6 \xi(1-6 \xi) z^{2}\right] \frac{\mathrm{d}}{\mathrm{d} \ln a}$. In the rest of the paper we will assume the function $\Gamma$ in the form $\Gamma(\lambda)=1-\frac{\alpha}{\lambda^{2}}$. In our approach the exact formulas for $w(z)$ parameterisations can be derived directly from the linearised solutions of the dynamical system in the vicinity of the critical points representing different epochs in the evolution of the universe (Table 1). In the special case of conformally coupled $(\xi=1 / 6)$ canonical $(\varepsilon=1)$ scalar field and dust matter $\left(w_{m}=0\right)$, they are as follows:

1) At the radiation domination epoch,

$$
\begin{gathered}
\left.w_{\mathrm{eff}}^{R}(z)\right|_{\xi=\frac{1}{6}}=\frac{1}{3}+\frac{2 \lambda_{1}^{*}}{3 \alpha} B_{1}\left(\frac{1+z}{1+z_{R}}\right)^{-1}+\left(\frac{1}{3} B_{1}^{2}-\frac{\alpha}{6} A_{1}^{2}\left(y_{1}^{(i)}\right)^{2}\right)\left(\frac{1+z}{1+z_{R}}\right)^{-2}+ \\
+\frac{1}{3} \lambda_{1}^{*} A_{1}\left(y_{1}^{(i)}\right)^{2}\left(\frac{1+z}{1+z_{R}}\right)^{-3}-\left(1+\frac{\alpha}{3}-\frac{\alpha}{6} A_{1} B_{1}\right)\left(y_{1}^{(i)}\right)^{2}\left(\frac{1+z}{1+z_{R}}\right)^{-4}- \\
-\frac{1}{3} \lambda_{1}^{*} B_{1}\left(y_{1}^{(i)}\right)^{2}\left(\frac{1+z}{1+z_{R}}\right)^{-5}-\frac{\alpha}{12} B_{1}^{2}\left(y_{1}^{(i)}\right)^{2}\left(\frac{1+z}{1+z_{R}}\right)^{-6}
\end{gathered}
$$

where $A_{1}=x_{1}^{(i)}-\frac{1}{\alpha}\left(\lambda_{1}^{(i)}-\lambda_{1}^{*}\right), B_{1}=x_{1}^{(i)}+\frac{1}{\alpha}\left(\lambda_{1}^{(i)}-\lambda_{1}^{*}\right),\left(\lambda_{1}^{*}\right)^{2}=\alpha^{2}$ and $z_{R}$ is the redshift of the radiation domination epoch.

2) At the matter domination epoch,

$$
\begin{aligned}
\left.w_{\mathrm{eff}}^{M}(z)\right|_{\xi=\frac{1}{6}}=-\alpha \frac{4}{3} A_{2}^{2}\left(y_{2}^{(i)}\right)^{2} & \left(\frac{1+z}{1+z_{M}}\right)^{-1}+\alpha \frac{8}{3} A_{2} B_{2}\left(y_{2}^{(i)}\right)^{2}\left(\frac{1+z}{1+z_{M}}\right)^{-3 / 2}- \\
& -\alpha \frac{4}{3} B_{2}^{2}\left(y_{2}^{(i)}\right)^{2}\left(\frac{1+z}{1+z_{M}}\right)^{-2}-\left(y_{2}^{(i)}\right)^{2}\left(\frac{1+z}{1+z_{M}}\right)^{-3}
\end{aligned}
$$

where $A_{2}=x_{2}^{(i)}+\frac{\lambda_{2}^{(i)}}{2 \alpha}, B_{2}=x_{2}^{(i)}+\frac{\lambda_{2}^{(i)}}{\alpha}$ and $z_{M}$ is the redshift of the matter domination epoch.

3) In the de Sitter state, we have two parameterisations whose type depends on the value of the parameter $\alpha$ characterising the shape of the function $\Gamma$.

- For $\alpha>-1 / 2$, we have a linear approach to the de Sitter state

$$
\begin{aligned}
& \left.w_{\mathrm{eff}}^{Q}(z)\right|_{\xi=\frac{1}{6}}=-1+\frac{1}{6 \Delta}(1-\alpha+\sqrt{\Delta}) A_{3}^{2}(1+z)^{3+\sqrt{\Delta}}+ \\
& +\left(\frac{\alpha}{\Delta} A_{3} B_{3}-2 y_{3}^{*}\left(y_{3}^{(i)}-y_{3}^{*}\right)\right)(1+z)^{3}+\frac{1}{6 \Delta}(1-\alpha-\sqrt{\Delta}) B_{3}^{2}(1+z)^{3-\sqrt{\Delta}}- \\
& -\frac{2 \alpha}{3 \Delta} A_{3}^{2} y_{3}^{*}\left(y_{3}^{(i)}-y_{3}^{*}\right)(1+z)^{6+\sqrt{\Delta}}+\left(\frac{4 \alpha}{3 \Delta} A_{3} B_{3} y_{3}^{*}-y_{3}^{(i)}+y_{3}^{*}\right)\left(y_{3}^{(i)}-y_{3}^{*}\right)(1+z)^{6}- \\
& -\frac{2 \alpha}{3 \Delta} B_{3}^{2} y_{3}^{*}\left(y_{3}^{(i)}-y_{3}^{*}\right)(1+z)^{6-\sqrt{\Delta}}- \\
& \quad-\frac{\alpha}{3 \Delta}\left(y_{3}^{(i)}-y_{3}^{*}\right)^{2}\left(A_{3}(1+z)^{\frac{\sqrt{\Delta}}{2}}+B_{3}(1+z)^{-\frac{\sqrt{\Delta}}{2}}\right)^{2}(1+z)^{9}
\end{aligned}
$$




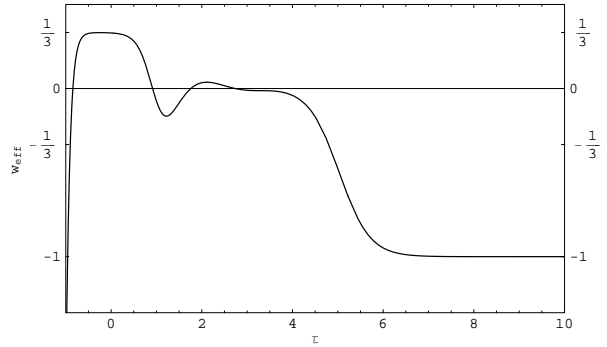

Fig. 1 The evolution of $w_{\text {eff }}$ given by the relation 8 for the nonminimally coupled canonical scalar field $\varepsilon=+1$ and the positive coupling constant $\xi$. The existence of a short time interval during which $w_{\text {eff }} \simeq \frac{1}{3}$ is the effect of the nonzero coupling constant $\xi$.

where $A_{3}=x_{3}^{(i)}+\frac{1}{2 \alpha}(3-\sqrt{\Delta}) \lambda_{3}^{(i)}, B_{3}=x_{3}^{(i)}+\frac{1}{2 \alpha}(3+\sqrt{\Delta}) \lambda_{3}^{(i)}$, and $\Delta=1+2 \alpha>0$.

- For $\alpha<-1 / 2$, we have a damped oscillatory approach to the de Sitter state

$$
\begin{aligned}
& \left.w_{\mathrm{eff}}^{Q}(z)\right|_{\xi=\frac{1}{6}}=-1-2 y_{3}^{*}\left(y_{3}^{(i)}-y_{3}^{*}\right)(1+z)^{3}+ \\
& +\frac{1}{3|\Delta|}\left(\left(C_{3}+\frac{|\Delta| \lambda_{3}^{(i)}}{2 \alpha}\right)^{2}-\alpha 4 C_{3}^{2}\right)(1+z)^{3} \sin ^{2}\left(\frac{\sqrt{|\Delta|}}{2} \ln (1+z)\right)+ \\
& +\frac{1}{3 \sqrt{|\Delta|}}\left(\left(C_{3}+\frac{|\Delta| \lambda_{3}^{(i)}}{2 \alpha}\right)\left(x_{3}^{(i)}+\frac{\lambda_{3}^{(i)}}{\alpha}\right)+2 C_{3} \frac{\lambda_{3}^{(i)}}{\alpha}\right)(1+z)^{3} \sin (\sqrt{|\Delta|} \ln (1+z))+ \\
& +\frac{1}{3}\left(\left(x_{3}^{(i)}+\frac{\lambda_{3}^{(i)}}{\alpha}\right)^{2}-\alpha\left(\frac{\lambda_{3}^{(i)}}{\alpha}\right)^{2}\right)(1+z)^{3} \cos ^{2}\left(\frac{\sqrt{|\Delta|}}{2} \ln (1+z)\right)- \\
& -\frac{4 \alpha}{3|\Delta|}\left(2 y_{3}^{*}\left(y_{3}^{(i)}-y_{3}^{*}\right)+\left(y_{3}^{(i)}-y_{3}^{*}\right)^{2}(1+z)^{3}\right) \\
& \quad\left(C_{3} \sin \left(\frac{\sqrt{|\Delta|}}{2} \ln (1+z)\right)-\frac{\sqrt{|\Delta|} \lambda_{3}^{(i)}}{2 \alpha} \cos \left(\frac{\sqrt{|\Delta|}}{2} \ln (1+z)\right)\right)^{2}(1+z)^{6},
\end{aligned}
$$

where $C_{3}=x_{3}^{(i)}+\frac{3 \lambda_{3}^{(i)}}{2 \alpha},|\Delta|=-1-2 \alpha>0$ and $z_{Q}=0$ is the redshift of the present time.

These parameterisations depend on three values $x_{n}^{(i)}, y_{n}^{(i)}, \lambda_{n}^{(i)}$ of the initial conditions for the linearised solutions at different epochs and the parameter $\alpha$ describing the shape of the function $\Gamma(\lambda)$. The common parameter $\alpha$ is present in all parameterisations and can be estimated from the observational data.

In this short note, we presented the possibility of extracting the equation of state parameterisations of dynamical dark energy directly from the dynamics of the underlying theoretical model. It is interesting that the obtained formulas for $w(z)$ are all that is needed for the realistic cosmological model, i.e., the radiation epoch required by the nucleosynthesis, matter domination phase and final acceleration epoch (see Fig. 1).

\section{References}

[1] M. Chevallier and D. Polarski, Int. J. Mod. Phys. D10, 213-224 (2001), arXiv:gr-qc/0009008

[2] E. V. Linder, Phys. Rev. D70, 023511 (2004), arXiv:astro-ph/0402503

[3] O. Hrycyna and M. Szydlowski, Phys. Lett. B651, 8-14 (2007), arXiv:0704.1651.

[4] O. Hrycyna and M. Szydlowski, Phys. Rev. D76, 123510 (2007), arXiv:0707.4471

[5] M. Szydlowski, O. Hrycyna, and A. Kurek, Phys. Rev. D77, 027302 (2008), arXiv:0710.0366

[6] A. Kurek, O. Hrycyna, and M. Szydlowski, Phys. Lett. B659, 14-25 (2008), arXiv:0707.0292.

[7] A. Kurek, O. Hrycyna, and M. Szydlowski, From model dynamics to oscillating dark energy parametrisation, arXiv:0805.4005.

[8] M. Szydlowski and O. Hrycyna, JCAP 01, 039 (2009), arXiv:0811.1493.

[9] O. Hrycyna and M. Szydlowski, Twister quintessence scenario, arXiv:0906.0335 